\title{
Evaluación de la técnica Western blot para la detección de antíge- nos de Hymenolepis nana
}

\author{
Evaluation of Western blot technique for detection of antigens of \\ Hymenolepis nana
}

Flora Chávez-Salas, Olenka Vásquez, Hermes Escalante

Departamento de Microbiología y Parasitología, Facultad de Ciencias Biológicas, Universidad Nacional de Trujillo, Av. Juan Pablo II s/n, Trujillo, Perú.

Email Flora Chávez-Salas:

fychs2003@yahoo.com

Email Olenka Vásquez:

olenka2004@hotmail.com

Presentado: $\quad$ 06/09/2006 Aceptado: $\quad$ 19/10/2007

\section{Resumen}

Este trabajo tuvo como objetivo evaluar la técnica de inmunoelectrotransferencia (Western Blot) para detectar los antígenos específicos de excreción/secreción de Hymenolepis nana en sueros de pacientes con himenolepiosis y con otras helmintiosis confirmadas. Se utilizó a Mesocricetus auratus "hamster" para obtener ejemplares adultos de H. nana. Los antígenos de excreción/secreción fueron obtenidos en el medio MEM (Minimum Essential Medium Eagle), y enfrentados con un grupo de sueros de pacientes con himenolepiosis confirmada para evaluar su calidad inmunológica y con sueros individuales de pacientes con himenolepiosis y con otras helmintiosis confirmadas para detectar mediante la técnica de "Western Blot", los antígenos específicos de este cestode. EI grupo de sueros de pacientes con himenolepiosis confirmada reconoció las bandas antigénicas de 50,$1 ; 42,6 ; 38,9 ; 32,9 ; 26,3 ; 22,4$ y $18,6 \mathrm{kDa}$; sin embargo, los sueros individuales reconocieron diferente número de bandas, siendo la de $50,1 \mathrm{KDa}$ la que fue reconocida por todos ellos. Los sueros de pacientes con helmintiosis confirmadas no reconocieron la banda de $50,1 \mathrm{kDa}$; $\sin$ embargo, dieron reacción cruzada con algunas de las demás bandas, a excepción de los sueros de pacientes con cisticercosis que no reconocieron a ninguna de las bandas de estos antígenos. Se concluye que el antígeno de excreción/secreción de $H$. nana de 50,1 kDa es específico de este cestode por ser reconocido por todos los sueros de pacientes con himenolepiosis confirmada y no por sueros de pacientes con otras helmintiosis utilizando la técnica de "Western Blot".

Palabras clave: Hymenolepis nana, Inmunoelectrotransferencia, Western blot, antígenos de excreción/secreción, himenolepiosis.

\begin{abstract}
Enzyme-linked immunoelectrotransfer blot assay or "Western blot" was evaluated to detect specific excretory/secretory antigens from Hymenolepis nana using sera from patient with hymenolepiosis and other confirmed helminthiosis. Hymenolepis nana adults were obtained from experimentally infected golden hamster, the parasits were cultured in Minimum Essential Medium Eagle to get excretory/secretory antigens which were faced to a pool of sera from patients with confirmed hymenolepiosis to evaluate its immunological quality and with individual sera from patients with hymenolepiosis and other confirmed helminthiosis to detect by mean "Western blot" technique, this parasite specific antigens. The pool of sera from patients with confirmed hymenolepiosis recognized seven antigenic bands; 50,$1 ; 42,6 ; 38.9 ; 26,3 ; 22,4$; and $18,6 \mathrm{kDa}$; however, the individual sera recognized different number of bands being 50,1 kDa band the most recognized by all of them. The sera from patients with confirmed helminthiosis did not recognized 50,1 KDa band; however they gave cross reaction with some of the other bands, except the sera from patients with cysticercosis which did not recognized any of this antigen bands. Consequently $50,1 \mathrm{kDa}$ antigen is specific because is recognized by all the sera from patients with confirmed hymenolepiosis and is not recognized by sera from patients with other helminthiosis using "Western blot" technique.
\end{abstract}

Keywords: Hymenolepis nana, immunoelectrotransfer blot, Western Blot, excretory/secretory antigens, hymenolepiosis.

\section{Introducción}

La himenolepiosis es una infección intestinal del hombre, las ratas y los ratones causada por cestodos del género Hymenolepis, siendo Hymenolepis nana o "tenia enana" la causante de la más frecuente de las cestodiasis humanas; esta parasitosis, habitualmente múltiple, afecta principalmente a niños, en quienes produce diversas manifestaciones clínicas con severa dificultad en el progreso ponderal (Atías, 1998).

La himenolepiosis, se encuentra con mayor frecuencia en países en vías de desarrollo, donde las condiciones socio-culturales y el nivel de saneamiento ambiental favorecen su propagación. En el Perú, la himenolepiosis es endémica y constituye un problema de salud pública, debido a las altas prevalencias que se presentan en muchas poblaciones rurales y suburbanas y por sus efectos de interés en poblaciones mal nutridas (Rodríguez y Calderón, 1991).

Las formas adultas de $H$. nana son una fuente de numerosos y potentes antígenos que inducen las correspondientes respuestas inmunes por parte del sistema linfático del hospedero; algunos de estos antígenos forman parte de las estructuras morfológicas del parásito y han sido denominados antígenos somáticos o estructurales, otros antígenos son producto de la actividad fisiológica del parásito y se los ha denominado de excreción/secreción, un gran número de los cuales son generalmente enzimas o catabolitos generados por el parásito vivo y en pleno desarrollo (Barriga, 1981).

Cada vez se otorga mayor importancia a los antígenos de excreción/secreción, ya que entre ellos se encuentran los compu- 
estos más específicos y las sustancias responsables de los fenómenos de protección del hospedero (Yarzabal, 1991). Por ello, se ha puesto mayor atención a estos antígenos, pues se ha comprobado que inducen una elevada respuesta inmune generándose, anticuerpos que poseen una especificidad tan precisa, capaz de distinguir entre diferentes cepas de una misma especie o entre distintos estadios evolutivos de un mismo parásito (Atías, 1998).

Para evaluar la inmunidad en las enfermedades parasitarias se emplean técnicas que se basan en la detección de antígenos específicos o de anticuerpos en diversos líquidos biológicos (Roitt et al., 1997). Una de las técnicas más importantes en el análisis y caracterización de fracciones antigénicas es la Electroinmunotransferancia (Western Blot), que básicamente es un proceso cualitativo que combina la selectividad de la electroforesis en gel de poliacrilamida, que permite fraccionar al antígeno, con la sensibilidad de la prueba inmunoenzimática, que pone en evidencia los complejos antígeno-anticuerpo formados (Garfín, 1990).

Querish et al., (1995) utilizando la técnica Western Blot, determinaron que el antígeno de $15 \mathrm{kDa}$ es específico de Fasciola hepatica al emplear sueros de pacientes con faciolosis; otros estudios han demostrado que los polipéptidos de 17, 23 y 28 kDa son específicos de este parásito (Díaz et al., 1998); en otra investigación se detectaron los antígenos de 43, 58, y $66 \mathrm{kDa}$ como específicos de la larva de Taenia solium (Ko y Ng, 1995).

Nuestra investigación se realizó en consideración de que la himenolepiosis, en el Perú, es una parasitosis de elevada frecuencia en niños en edad pre-escolar y escolar, y que no se han efectuado investigaciones relacionadas con la caracterización de antígenos específicos de excreción/secreción de H. nana, los cuales podrían servir en estudios de inmunodiagnóstico o en la elaboración de vacunas.

En el presente trabajo se determina la eficacia de la técnica de Inmunoelectrotransferencia o Western Blot en la detección de antígenos específicos de excreción/secreción de $H$. nana utilizando sueros de pacientes con himenolepiosis y con otras helmintiosis confirmadas.

\section{Material y métodos \\ Sueros}

Se obtuvieron 42 sueros de pacientes con parasitosis confirmada; de los cuales: 18 procedieron de alumnos del Centro Educativo Simón Bolivar de Otuzco, La Libertad; de estos sueros 12 procedieron de pacientes con himenolepiosis, cuatro con difilobotriosis y dos con ascariosis. Los 24 sueros restantes procedieron de la seroteca del Centro de Análisis e Investigación "Escalabs" E.I.R.L de Trujillo-Perú, de los cuales, siete eran de pacientes con cisticercosis, seis con hidatidosis, seis con taeniosis por Taenia solium, tres con taeniosis por Taenia saginata y dos de recién nacidos libres de infección por parásitos que sirvieron como control negativo.

\section{Antígenos}

Para la obtención de los antígenos de $H$. nana se infectaron 30 ejemplares de $M$. auratus "hamster" vía oral con huevos viables de $H$. nana obtenidos de las heces de un grupo de niños con himenolepiosis confirmada. Los hamsters se sacrificaron a los 21 días para extraer los ejemplares adultos de este cestote, los que fueron cultivados asépticamente en el Medio Mínimo Esencial Eagles. El medio con los antígenos de excreción/secreción de $H$. nana de cada cultivo se recolectó a las ocho, nueve, 14, 15 y 18 horas de incubación. La concentración de proteínas presentes en el medio con los antígenos se determinó por el método colorimétrico de Bradford (Bradford, 1976).

La preparación de los reactivos y la ejecución de la técnica se realizaron siguiendo el manual de Tsang (1986). Los antígenos se prepararon a la concentración de $0,025 \mu \mathrm{g} / \mu \mathrm{l}$, para lo cual fueron tratados con $0,01 \mathrm{M}$ de Tris- $\mathrm{HCl}$ a $\mathrm{pH} 8,0,1 \%$ de dodecil sulfato de sodio (SDS), $6 \%$ de glicerol y $0,01 \%$ de azul de bromofenol. Se calentó a $65^{\circ} \mathrm{C}$ por 20 minutos.

Los antígenos de excreción/secreción fueron colocados en los pocillos en una cantidad equivalente a $1 \mu \mathrm{l}$ por cada milímetro de ancho del gel. Los corridos fueron hechos en minigeles de 8x7 x0,05 $\mathrm{cm}, 12 \%$ de poliacrilamida y $200 \mathrm{~V}$. La transferencia al papel de nitrocelulosa se hizo utilizando un buffer de transferencia $(0,2$ $\mathrm{M}$ Tris/ $\mathrm{HCl} \mathrm{pH} 8,0 ; 20 \%$ metanol y agua destilada) y a $1 \mathrm{~A}$ por espacio de una hora. Las láminas de nitrocelulosa con los antígenos separados fueron cortadas en tiras de $3 \mathrm{~mm}$ de ancho.

Cada uno de los sueros fue enfrentado con los antígenos de excreción/secreción de $H$. nana; para lo cual cada uno de los sueros fueron diluidos a 1/60 en una solución de PBS Tween 20 y leche descremada al 5\%, como bloqueador de las zonas ocupadas por los antígenos. El conjugado enzimático (Anti Ig G marcada con Peroxidasa) se usó por la dilución de 1/1000 siendo reveladas las bandas con solución de sustrato $\left(\mathrm{H}_{2} \mathrm{O}_{2}\right.$ al $0,01 \%$ ) y la diaminobenzidina a la concentración de $0,5 \mathrm{mg} / \mu \mathrm{l}$ por 5 a 10 minutos.

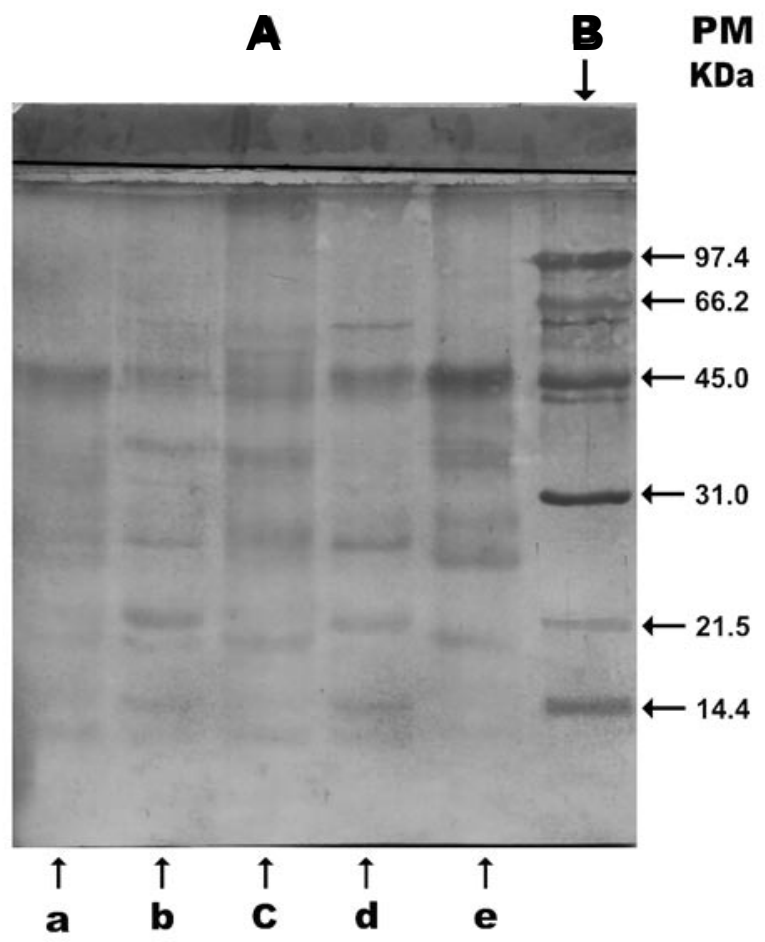

Figura 1. Bandas de antígenos excreción/secreción de Hymenolepis nana detectadas por "Western blot", utilizando un grupo de sueros de pacientes con himenolepiosis confirmada. (A) Antígenos de excreción /secreción de Hymenolepis nana obtenidos de diferentes cultivos en Minimum Essential Médium Eagle a los tiempos de incubacion de 8 (a), 9 (b), 14 (c), 15 (d) y 18 (e). (B) Marcador de peso molecular (PM) en Kilodaltons $(\mathrm{kDa})$. 


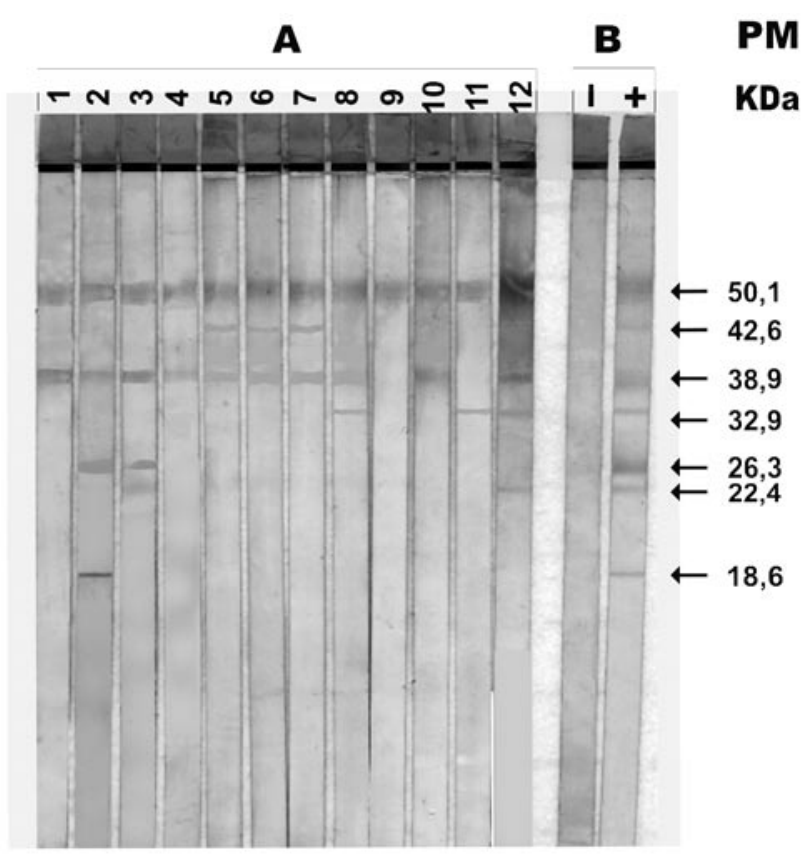

Figura 2. Bandas de los antígenos de excreción /secreción de Hymenolepis nana detectados por la técnica de "Western blot" utilizando sueros individuales de paciente con himenolepiosis confirmada. (A) sueros de pacientes con himenolepiosis confirmada. (B) Sueros controles.

\section{Resultados}

El porcentaje de infección experimental de Mesocricetus auratus "hamster", por Hymenolepis nana fue de $67 \%$, con un promedio de 40 ejemplares adultos de $H$. nana por hamster infectado, los cuales llegaron a medir entre uno y cinco centímetros de longitud.

La calidad inmunológica de los antígenos de excreción/secreción de $H$. nana obtenidos de diferentes cultivos en el medio MEM (Minimum Essential Médium Eagle) a las ocho, nueve, 14, 15 y 18 horas de incubación se evaluó enfrentándolos con un pool de 12 sueros de pacientes con himenolepiosis confirmada mediante la técnica de Western Blot reconociéndose siete bandas antigénicas cuyos pesos moleculares fueron: 50,1; 42,6; 38,9; 32,$9 ; 26,3 ; 22,4$ y $18,6 \mathrm{kDa}$, siendo los antígenos obtenidos a las 18 horas, los que mostraron mayor nitidez (Fig. 1.)

Los antígenos de $H$. nana obtenidos a las 18 horas de incubación en medio MEM Eagle, fueron enfrentados con 12 sueros individuales de pacientes con himenolepiosis confirmada, encontrando que cada suero reconoció diferente número de bandas antigénicas siendo la de $50,1 \mathrm{kDa}$, la que fue reconocida por todos ellos. (Fig. 2.).

Los antígenos específicos de excreción/secreción de $H$. nana obtenidos a las 18 horas de incubación en medio MEM Eagle, se evaluó utilizando sueros individuales de siete pacientes con cisticercosis, seis con hidatidosis, seis con taeniosis por Taenia solium, tres con taeniosis por Taenia saginata, cuatro con difilobotriosis y dos con ascariosis, encontrando que ninguno de los sueros de pacientes con estas parasitosis reconoció la banda de 50,1 kDa pero si dieron reacción cruzada con algunas de las demás bandas, a excepción de los sueros de pacientes con cisticercosis que no reconocieron a ninguna de las bandas de estos antígenos (Fig. 3).

\section{Discusion}

Se logró el desarrollo de las formas adultas de Hymenolepis nana en hamster aún sin ser este su hospedero natural (Smyth, 1969; Barriga, 1981, Monroy, 1992). Esto fue posible con el empleo de acetato de metilprednisolona que inmunodeprimió a los animales y permitió la infección experimental, favoreciendo no sólo al establecimiento del parásito sino también al desarrollo del mismo, hasta alcanzar su forma adulta con producción de huevos.

Por otro lado el porcentaje de infección experimental de 67\%, se debe probablemente, al hecho de que el hámster al no ser hospedero natural de $H$. nana, presenta condiciones físico-químicas en su tracto intestinal que no favorecen el establecimiento de este cestode. Además, la obtención de un promedio de 40 ejemplares del parásito por hámster infectado, cuyo número puede con-

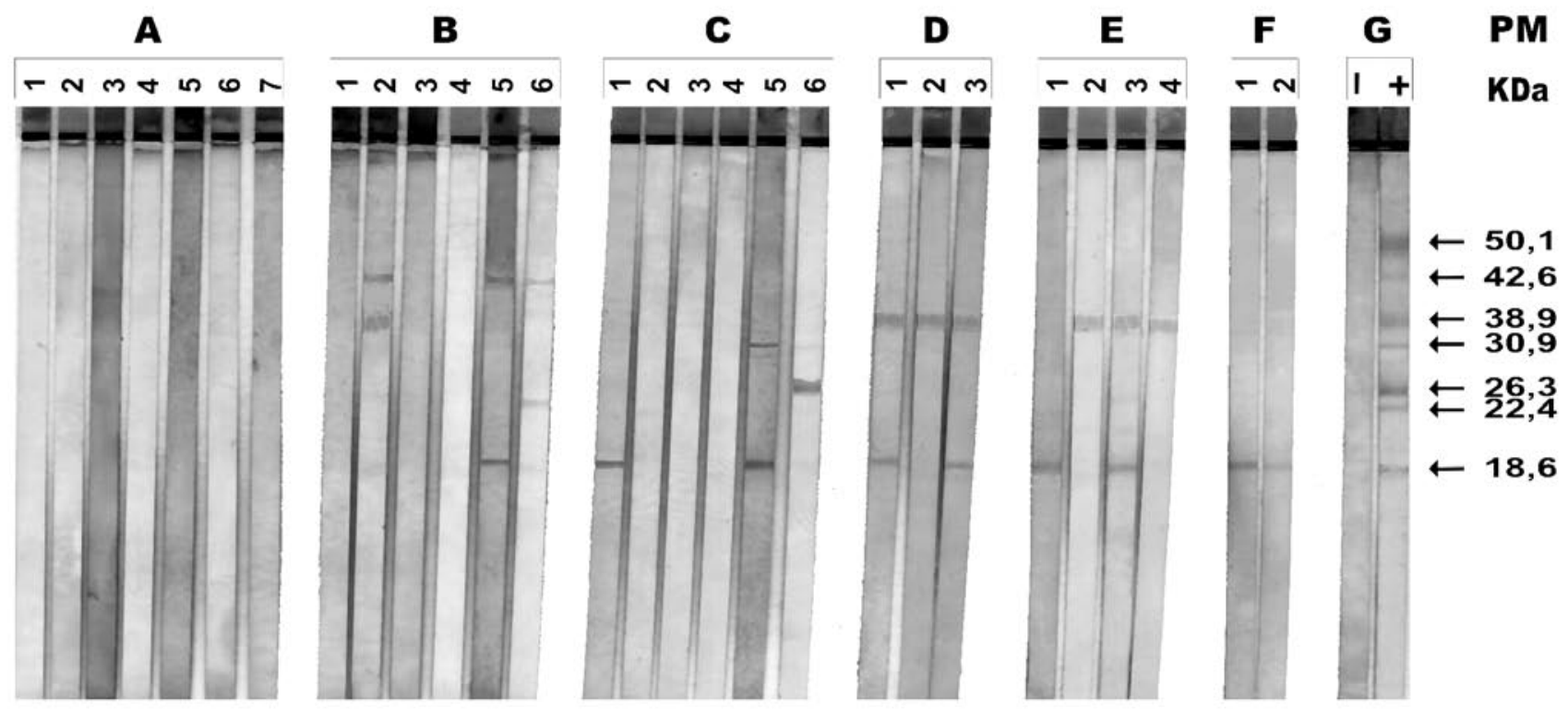

Figura 3. Bandas de los antígenos de excreción /secreción de Hymenolepis nana detectados por la técnica de "Western blot" utilizando sueros individuales de paciente de pacientes parasitados por helmintos intestinales. (A) Con cisticercosis. (B) Con hidatidosis. (C) Con taeniosis por Taenia solium. (D) Con taeniosis por Taenia saginata. (E)Con difilobotriosis. (F) Con ascariosis. (G) Sueros controles. 
siderarse no elevado, puede deberse a que el número de vermes varía en relación inversa al tamaño en el intestino delgado del hospedero (Maldonado, 1965).

La técnica de Western Blot tiene elevada sensibilidad, ya que detecta los complejos antígeno-anticuerpo mediante la técnica de Elisa y también elevada especificidad, debido a que fracciona los componentes proteínicos del antígeno. En el presente trabajo se optó por utilizar los antígenos en estado puro, por la baja concentración en que se encontraban, debido al pequeño tamaño de $H$. nana y la poca excreción de proteínas al medio MEM Eagle.

El empleo de los antígenos de excreción/secreción de H. nana obtenidos a las 18 horas de cultivo se debe a la mayor nitidez y menor número de bandas inespecíficas observadas en comparación con los antígenos obtenidos a las ocho, nueve, catorce y quince horas de cultivo. Esto podría atribuirse al número y tamaño de los ejemplares de $H$. nana cultivados por tubo así como a la calidad de los antígenos producidos por los mismos.

El número de bandas y sus respectivos pesos moleculares encontrados en el presente trabajo, no pueden ser comparados con otros trabajos por ser este el primero en su género con antígenos de excreción/secreción de $H$. nana.

El patrón de respuesta observado en cuanto al número de bandas e intensidad de la reacción indica que existe heterogeneidad de la respuesta inmunológica producida por los anticuerpos del hospedero contra el parásito (Vaz et. al., 1997; Larralde et.al., 1989)

La banda de 50,1 kDa, considerada como antígeno específico de $H$. nana, no mostró reacción cruzada con los anticuerpos de los sueros de pacientes con otras parasitosis, lo que indica que se trata de un producto metabólico propio de este parásito y que los anticuerpos formados contra los antígenos de excreción/secreción son capaces de distinguir entre los distintos estadíos evolutivos de un mismo parásito y entre diferentes parásitos del mismo género familia y orden (Atías, 1998).

De los siete sueros confirmados a cisticercosis, ninguno reaccionó con las siete bandas antigénicas mencionadas, no encontrándose reacción cruzada con esta zoonosis, resultados que concuerdan con los obtenidos por Díaz et. al., (1992) quienes atribuyen la ausencia de reacción a la falta de anticuerpos contra $H$. nana en el suero de los pacientes. Díaz et. al., (1992) tambien explica que las reacciones cruzadas observadas entre los sueros de pacientes con otras helmintiasis intestinales y los antígenos de $H$. nana, se deben a la proximidad filogenética entre estos parásitos. La existencia de reacciones cruzadas indicaría también que estos parásitos presentan algunos componentes antigénicos comunes con $H$. nana que dan lugar a estas reacciones.

La reacción positiva observada en la banda de $18,6 \mathrm{kDa}$ del antígeno de excreción/secreción de $H$. nana con los sueros de pacientes con ascariosis, probablemente no se deba a una reacción cruzada, sino a que estos pacientes, además de presentar ascariosis quizás hayan presentado himenolepiosis por proceder de una zona endémica.

\section{Literatura citada}

Atías, A. 1998. Parasitología Clínica. 3ra Ed. Editorial Mediterráneo. Santiago - Chile.

Barriga, O. 1981. The Inmunology of Parasitic Infections. A handbook for physicians, veterinarians and biologists. University Park Press. Baltimore, Maryland.

Bradford, M. 1976. A rapid sensitive method for the quantification of microgram quantities of protein utilizing the principle of protein dye binding. Analytical Biochemistry. 72:248-254.

Díaz, J.; M. Verastegui; R. Gilman; C. Victor; J. Tsang; B. Joy; C. Pilcher; C. Gallo; H. García; P. Torres; T. Montenegro; E. Miranda \& the cysticercosis working group in Peru. 1992. Inmunodiagnosis of human cysticercosis (Taenia solium): A field comparison of an antibody-enzyme-linked inmunosorbent assay (Elisa), Inmunoelectrotransfer Blot (EITB) assay in Peru. Am. J. Trop. Med. Hyg. 46(5): 610-15.

Díaz, A.; O. Li Elías; O. Otero. Otero; C. García \& A. Espino. 1998. Identificación mediante Western Blot de inmunñogenos de Fasciola Hepática reconocidos por los sueros de ratas infectadas experimentalmente. Rev. Cubana de Med. Trop. 50(1):12.

Garfín, D. 1990. Isoelectric focusing. Methods Enzymol 182:459-477

Larralde, C.; R. Montoya; E. Sciutto.; M. Díaz; T. Govezensky \& E. Coltorti, 1989. Deciphering Western Blot of tapeworms antigens (Taenia solium, Echinococcus granulosus and Taenia crassiceps) reacting with sera from neurocysticercosis and hydatid disease patients. Am. J. Trop. Hyg. 40: 282-90.

Ko, R \& T. Ng. 1998. Evaluation of excretory/secretory products of larval Taenia solium as diagnostic antigens for porcine and human cisticercosis. University of Hong Kong. China. Journal of Helmintology. 72(2): 147-154.

Maldonado, J. 1965. Helmintiasis del hombre en América. Editorial Científico Médica. Barcelona. España.

Monroy, A.; J. Gómez; A. Ramírez \& G. Carrillo. 1992. Reconocimiento por inmunotransferencia de antígenos de Taenia solium y su larva. Rev. Lat-Amer. Microbiol. 34: 33-38.

Qureshi, T.; G. Wagner; D. Drawe; D. Davis. \& T. Craig. 1996. Enzyme-linked inmunotransfer blot análisis of excretorysecretory proteins of Fascioloides magna and Fasciola hepática. Vet Parasitol. 58(4): 357

Rodríguez, J. \& J. Calderón. 1991. Parasitismo intestinal en niños en edad pre-escolar de Tarapoto. Revista de Gastroenterología del Perú. 11(3):153-60

Roitt A; Brostoff J. \& Male, D. 1997. Inmunología. 4ta. ed. Edit. Harcourt-Brace. Madrid-España

Smyth, J. 1969. The Physiology of the Cestodes. Constatable LTD. Hopctoun Street, Edinmburgh 7.

Tsang, V. 1986. Enzyme Linked Inmunotransfer Blot technique (Western Blot) for human T Lynphotopic Virus type III/Lynphoadenopathy-associated Virus (Htlv/LAV) Antibodies. US Department of Health and Human services. Public Health Service Centers for Disease Control. Atlanta. Inmunology series $\mathrm{N}^{\circ} 15$. Procedural guide.

Vaz, A.; C. Nunes; R. Piazza; J. Livramento; M. Da Silva \& P. Nakamura. 1997. Inmunoblot with cerebrospinal fluid from patients with neurocysticercosis using antigen from cysticerci of Taenia solium and Taenia crassiceps. Am. J. Trop Med. Hyg. 57: 354-7

Yarzabal, L. 1991. Aspectos inmunológicos de las enfermedades parasitarias. Inmunología Clínica. Venezuela. 2:7-21. 